\title{
Tangible Interfaces to Digital Connections, Centralized versus Decentralized
}

\author{
Matthijs Kwak, Gerrit Niezen, Bram van der Vlist, Jun Hu, and Loe Feijs \\ Designed Intelligence Group, Department of Industrial Design \\ Eindhoven University of Technology \\ Den Dolech 2, 5612AZ, Eindhoven, The Netherlands
}

\begin{abstract}
In the era of distributed digital media, technology is moving to the background and interoperability between devices increases. The handles for users to explore, make and break connections between devices seem to disappear in overly complex menu structures displayed on small screens. Two prototypes have been developed that introduce a tangible approach towards exploring, making and breaking connections between devices in a home environment. Findings suggest that users are better able to project their mental model of how the system works on decentralized representations and that a tangible solution is not necessarily a better one.
\end{abstract}

Keywords: Ontology, semantic connections, tangible user interface, internet of things.

\section{Introduction}

In the era of distributed digital media, especially in a home environment, devices are connected to one another to create preferred experiences. A home theatre system is one example of how multiple devices can create one joint experience when interoperating 5637. With the introduction of portable media players, possibilities and needs for content sharing are even bigger. Currently these devices are connected wirelessly or with all kinds of cables, and users are currently occupied with finding the right cables to connect devices and have to deal with cables that physically allow for connections that are not possible. Even more, some possible connections never get explored, simply because physical cables do not allow for it. Wireless technologies such as Bluetooth solve part of the problem, but introduce overly complex menu structures and devices without proper interfaces. A single task like sharing music from the one device to another currently involves multiple steps on both devices, while one single high-level effort would be desirable.

In 'The Internet of Things' 8, and 'Shaping Things' [14] a world is sketched in which each everyday object has an unique identity and is connected to the internet. In this world, technology has moved to the background and interoperability between devices has been achieved. Provided that these devices are able to communicate with each other and with the user, this could mean the end of

Z. Pan et al. (Eds.): Transactions on Edutainment V, LNCS 6530, pp. 132-146, 2011.

(C) Springer-Verlag Berlin Heidelberg 2011 
compatibility problems and the hassle of using cables, and that users will have less physical and visual handles to make sense of their environments and the devices therein. Design can play an important role in this sense-making with paradigms like Tangible User Interfaces 4], that believe that physical handles for digital information provide users with more freedom and control.

The SOFIA project is a European research project that targets to "make 'information' in the physical world available for smart services - connecting the physical world with the information world" 13 . Within this project a "Semantic Connections" demonstrator was developed named Interaction Tile. This demonstrator allows users to tangibly explore, make and break connections between devices in a smart home environment [11617.

A second demonstrator, named Interaction Tabs, was developed to explore alternative possibilities of Tunis. Where the Interaction Tile provides users with a centralized way of exploring, making and breaking connections, the Interaction Tabs provides users with a decentralized way to perform the same tasks.

In order to see which demonstrator would be the easiest to use and allow for a better projection of the users' mental model, a user experiment was set up to answer the following questions:

- Are the demonstrators a better alternative, compared to the conventional method?

- Will the users be able to work equally well with both demonstrators?

In the first question, "better" is in the sense that exploring, making and breaking connections are easier (more efficient) and more satisfactory (positive user experience). An important aspect is the mental model that the participants have and how it compares to the actual architecture of the system.

\section{Background}

\subsection{SOFIA Project and the Interaction Tile}

SOFIA (Smart Objects for Intelligent Applications) is a European research project addressing the challenge of Artemis sub-programme 3 on Smart Environments. The overall goal of this project is to connect the physical world with the information world, by enabling and maintaining interoperability between electronic systems and devices. Our contribution to the project is to develop smart applications for the smart home environment, and to develop novel ways of user interaction. For users to truly benefit from smart environments, it is necessary that users are able to make sense of such an environment. One way of facilitating this "sense making" is through design. Our contribution to the SOFIA project aims at developing theories and demonstrators, and investigating novel ways of user interaction with the smart environment, through interaction with smart objects in the space.

To illustrate the concepts and ideas developed in the project, a demonstrator was developed. The demonstrator is a tile-like interactive object that allows for both exploration of a smart space in terms of connections, and manipulation of 


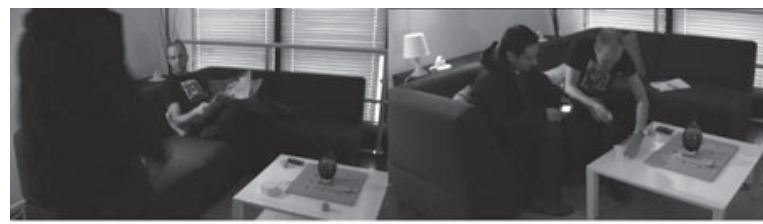

...and wants to play some of his music Dries visits Mark with his music player... on Mark's sound system

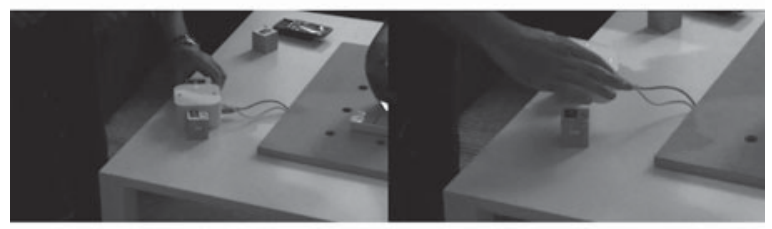

they explore connection possibillities

between music player and sound system they make the connection

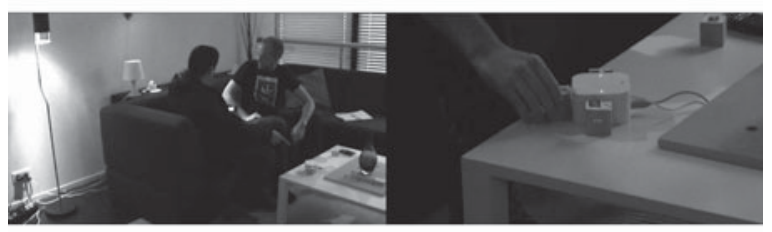

they also want to connect the

....and explore whether the ambient ambient lighting system...

lighting system can be connected as well

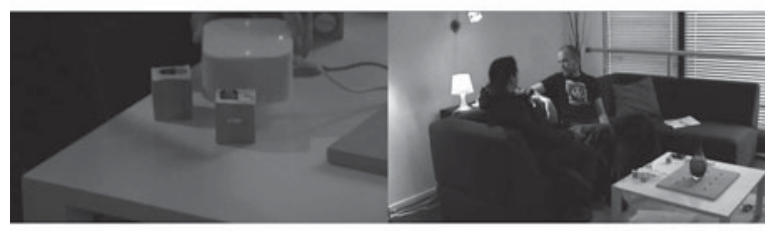

they make the connection...

...and enjoy the music and lighting effects

Fig. 1. Interaction Tile in action

these connections and information/data streams through direct manipulation. This is done by making simple spatial arrangements. The Interaction Tile visualizes the various connections, by enabling users to explore which objects are connected to one another, and what can be connected to what. Colored LED lighting and light dynamics visualize the connections and connection possibilities between the devices, by means of putting devices close to one of the four sides of the tile. A user can then check whether there is a connection and if not, whether a connection is possible. By simply picking up the tile and shaking it, a user can make or break the connection between the devices present at the interaction tile.

Fig. [1 shows a use case example of how the interaction tile can be used. The Interaction Tile supports the following interactions:

- viewing/exploring existing connections;

- viewing/exploring connection possibilities;

- making connections;

- breaking connections. 
We want to enable users to explore and manipulate the connections within the smart space without having to bother with the lower-level complexity of the architecture. We envision this "user view" to be a simplified view (model) of the actual architecture of the smart space. Conceptually, the connections are carriers of information; in this case they carry music. Depending on the devices' capabilities (e.g. audio/video input and/or output) and their compatibility (input to output, but no output to output), the Interaction Tile will show the connection possibilities. In our current demonstrators we do not distinguish between different types of data since we are only dealing with audio, but it will be inevitable in more complex scenarios. The Interaction Tile acts as an independent entity, inserting events and data into a triple store and querying when it needs information. The different types of events and the connections between smart objects and their related properties are described in an ontology. The ontology with "isa" relationships indicated is shown in fig. 2, Fig. 3 shows the architecture of the current setup.

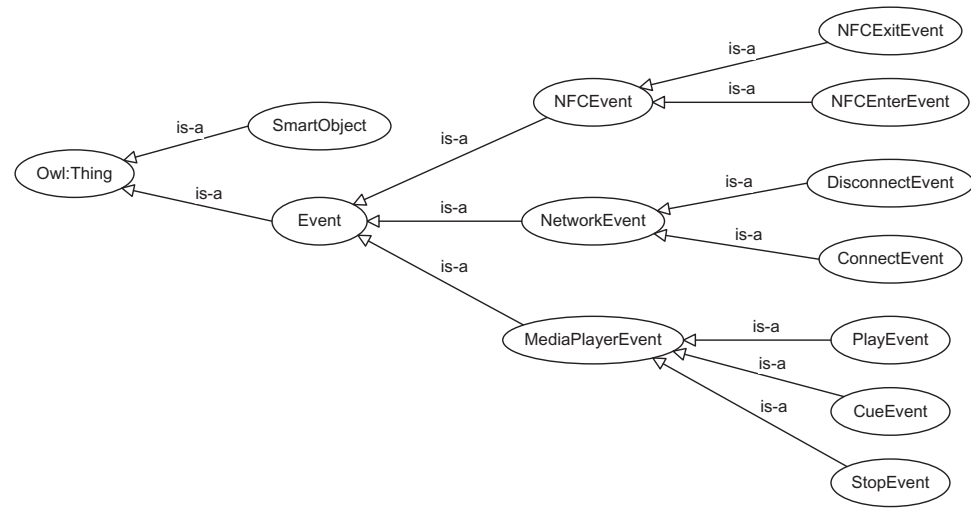

Fig. 2. Ontology indicating "is-a" relationships

We implemented the demonstrator using the Jena Semantic Web framework, the Processing library for Java, and Python for S60. Every interaction with either the music players (smart phones) or the interaction tile results in an interaction event. A semantic reasoner (Pellet) is used to reason about these low-level events in order to infer higher-level results.

When the user shakes the tile to establish a connection, two NFCEnter Event events (generated by the RFID reader inside the interaction tile) by two different devices that are not currently connected, will result in a new connectedTo relationship between the two devices. Because connectedTo is a symmetric relationship, the reasoner will automatically infer that a connection from device $A$ to device $B$ means that device $B$ is also connected to device $A$. Since connectedTo is also an irreflexive property, it is not possible for a device to be connected to itself. A generatedBy relationship is also created between the event and the smart device that generated it, along with a timestamp and other event metadata. 


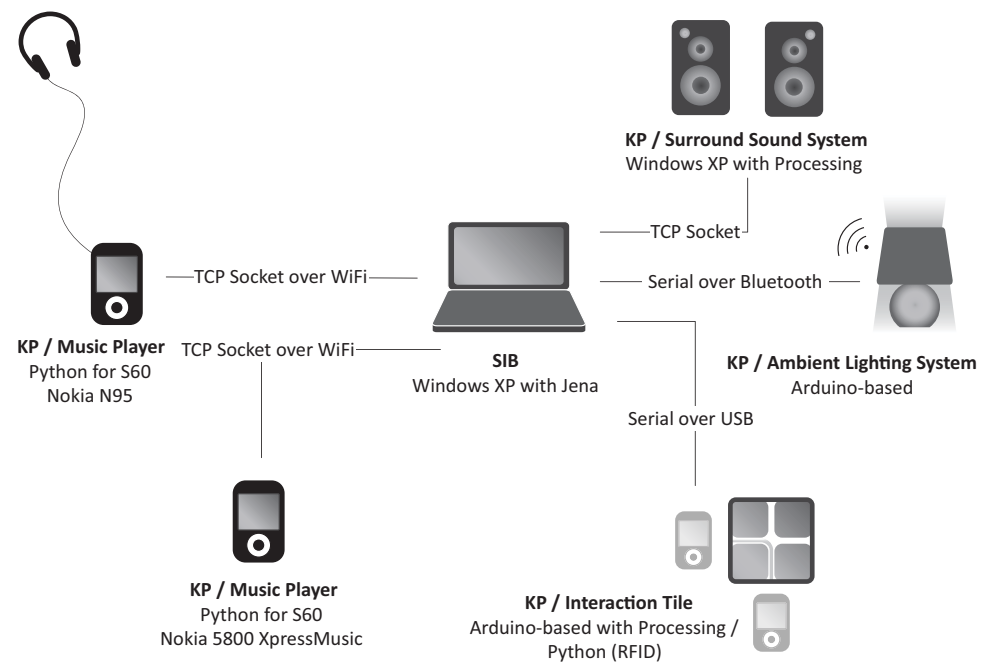

Fig. 3. Overview of the demonstrator

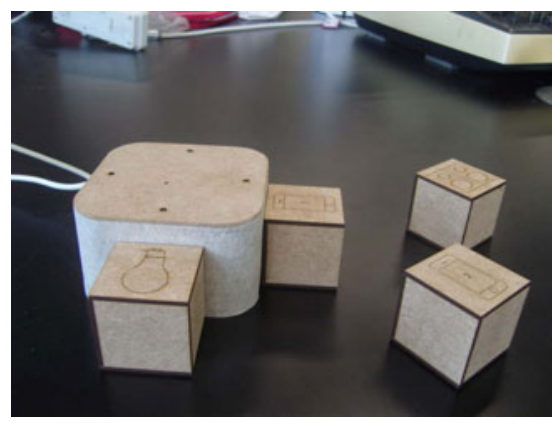

(a) Interaction Tile

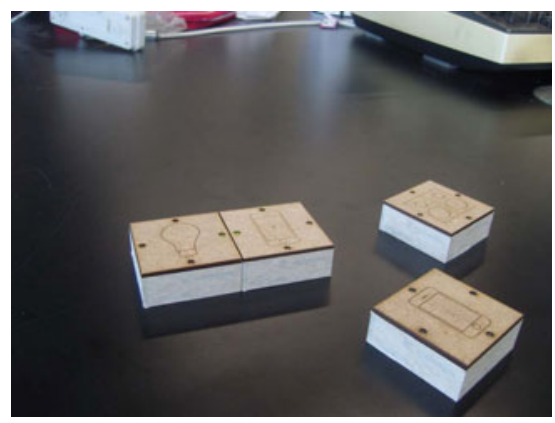

(b) Interaction Tabs

Fig. 4. Two versions of the demonstrator

\subsection{Interaction Tabs: Decentralized}

A second demonstrator was developed to explore other tangible solutions. The Interaction Tabs demonstrator was implemented using the same set-up and software, but replacing the Interaction Tile (fig. 4(a) with the Interaction Tabs (fig. 4(b)).

First the Interaction tile was analyzed using the Frogger Framework (fig. [5) 18. The Frogger framework is a design framework that allows for both analyzing and synthesizing interactions. Six relations (couplings) between action and reaction are described:

Time. The product's reaction and the user's action coincide in time.

Location. The reaction of the product and the action of the user occur in the same location. 


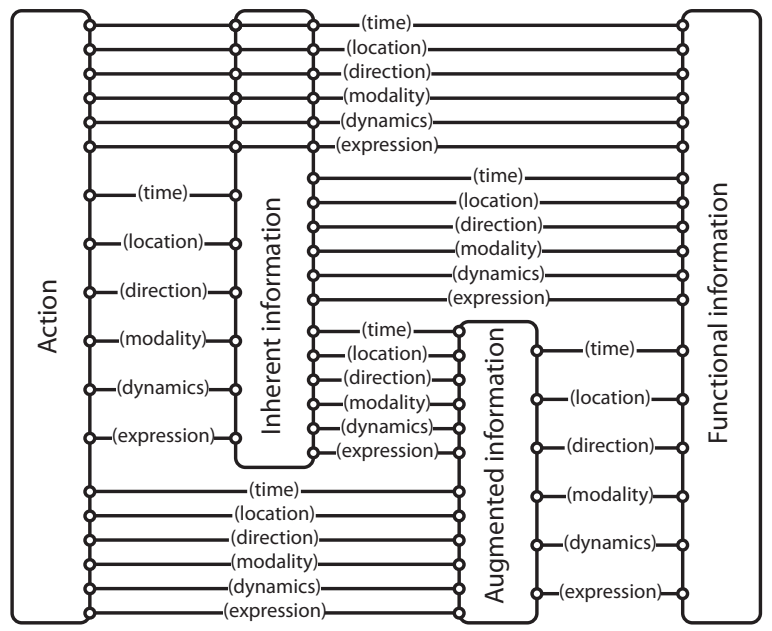

Fig. 5. Frogger Framework [18]

Direction. The direction or movement of the product's reaction (up/down, clockwise, right/left and towards/away) is coupled to the direction or the movement of the user's action.

Dynamics. The dynamics of reaction (position, speed, acceleration, force) is coupled to the dynamics of the action (position, speed, acceleration force).

Modality. The sensory modalities of the product's reaction are in harmony with the sensory modalities of the user's action.

Expression. The expression of the reaction is a reflection of the expression of the action.

Furthermore, Wensveen distinguishes between three types of feedback and feedforward; functional, augmented and inherent [18. Feedback is "the return of information about the result of a process or activity" [2. Functional feedback is "the information generated by the system when performing its function". Augmented feedback is information generated by an additional source, not directly related to the system and its function. Inherent feedback was defined by Laurillard 9] as "information provided as a natural consequence of making an action. It is feedback arising from the movement itself." Feedforward is the information provided to the user before any action has taken place. Inherent feedforward communicates what kind of action is possible and how one is able to carry this action out. When an additional source communicates what kind of action is possible it is considered augmented feedforward. Functional feedforward communicates the more general purpose of a product.

There are many improvements one can consider for the Interaction Tile (fig. 6) when putting it in the Interaction Frogger framework. We decided, though, to stay as close to the original design as possible; for research purposes it is best to change as little as possible in order to be able to clearly identify what exactly causes change in user behavior (if users' behavior actually changes). 


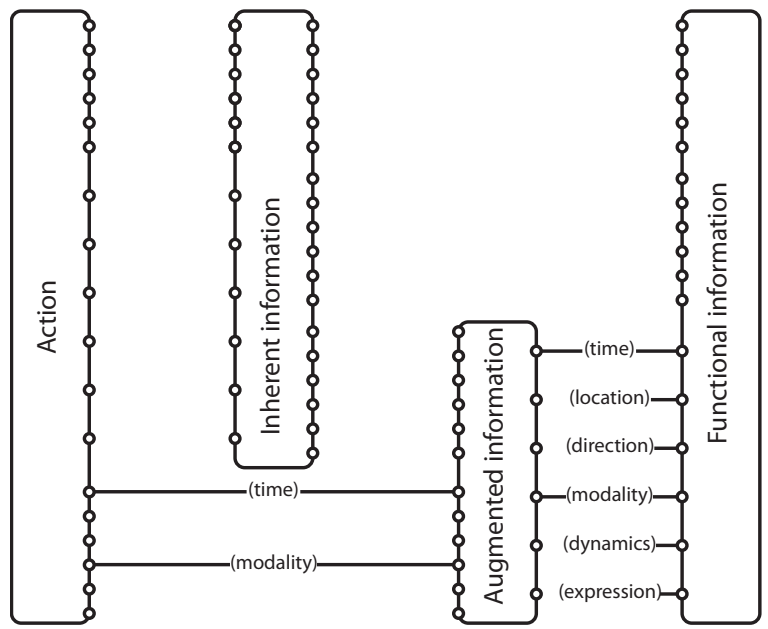

Fig. 6. Interaction Tile in the Frogger Framework

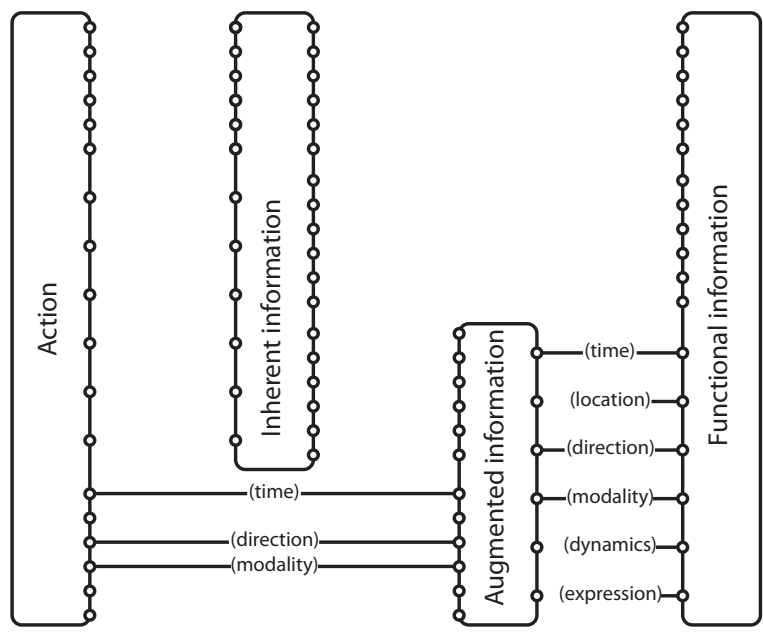

Fig. 7. Interaction Tabs in Frogger Framework

By removing the center tile and moving its functionality into the cubes that represent the devices being connected/disconnected, the demonstrator would become simpler as this would allow for a more direct manipulation of the connections. The (digital) states of the connections would be physically represented, cubes being aligned means that the devices that they represent are connected, and not being aligned means they are disconnected.

Inspired by Siftables [10] the cubes were transformed to tabs, because tabs have a clear top and bottom. This does still afford stacking, but hopefully users would understand that the tabs were to be aligned (fig. 4(b)). An LED at each side gives the feedback: 
Red. No connection possible. This occurs when no relation is possible between the two devices of which the tabs are aligned.

Green. This occurs when a relation exists between two devices of which the tabs are aligned.

To make a connection, the tabs that represent these devices have to be aligned. To break the connection, the alignment has to be broken.

As a result of removing the center tile, the Interaction Tabs will no longer allow for the exploration of existing connections and connection possibilities without immediately manipulating the connections. Moving towards having a more physical approach might influence the scalability and have some other practical implications; however, this is beyond the scope of this paper as we try to focus on the interaction itself.

We expect these differences to also have different influences on the user's mental model, in the way users conceptualize connections (including properties like: persistence, transitivity and directionality) and differences in how they imagine devices to be connected, e.g. devices connected in a networked fashion versus connecting devices peer-to-peer. When we also analyze the Interaction Tabs using the Interaction Frogger framework (fig. 7), there are two ways of comparing the two demonstrators:

First we consider the Interaction Tile and Interaction Tabs as being part of the same demonstrator set-up as shown in Fig. 3, serving as a device to manipulate the connections between the various devices in the set-up. The changes might improve the interaction with regard to:

Direction. With the center tile removed, the direction of making and breaking connections (although done remotely) corresponds better.

Modality. With the shaking interaction removed, the modality of making and breaking connections corresponds better.

Secondly, we look at the interaction devices themselves as if they were standalone products. This reveals more improvements: Information about time, location, direction and modality are augmented and inherent when the center tile and shaking interaction are removed. For the Interaction Tile only location is inherent, and time, direction and modality are augmented (fig. 8 and fig. 9).

\section{Experiment}

In order to answer the questions raised in the beginning of this paper, an experiment was conducted.

\subsection{Participants}

12 participants were invited to the experiment, in which 3 were women and 9 were male. The participants were between 21 and 26 years old. All but one had a BSc. in Industrial Design. One also had a MSc. in Industrial Design. 


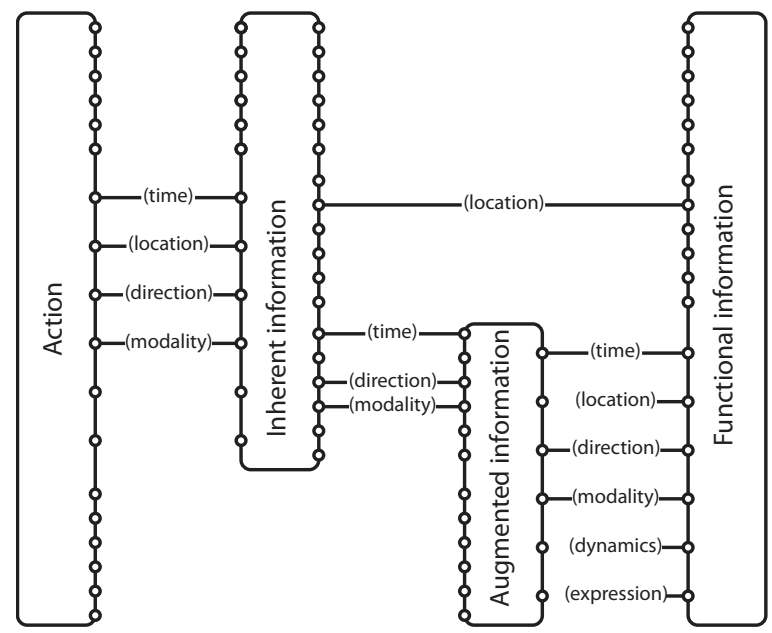

Fig. 8. Interaction Tile in the Frogger Framework (stand-alone)

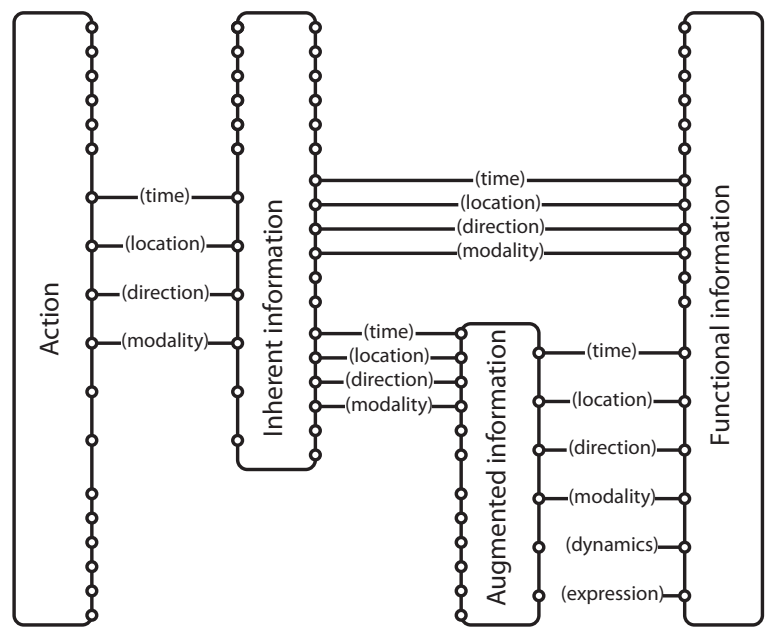

Fig. 9. Interaction Tabs in Frogger Framework (stand-alone)

\subsection{Apparatus}

The following equipment was used:

- Dell laptop (Windows XP) with Wi-Fi, Bluetooth antenna, audio out and two USB ports.

- Nokia N95 mobile phone with Python installed, running a script to be able to play a sample and communicate with the laptop.

- Nokia 5800 XpressMusic mobile phone with Python installed, running a script to be able to play a sample and communicate with the laptop. 
- Ambient Light lamp: A Bluetooth Arduino-based lamp that renders the music in colored lighting using RGB LEDs, with code running to be able to communicate with the laptop.

- Samsung NV8 digital camera mounted on a tripod to record the experiment.

- Philips speaker set with two satellite speakers and a subwoofer, connected to the Dell laptop.

- Netgear WPN824 wireless router.

- Interaction Tile and Interaction Tabs, including software in Java and Python.

A controlled setting was used to conduct the tests. The study took place in the 'Contextlab' at Eindhoven University of Technology. The lab is furnished to look like a living room, which is the context in which the demonstrators would normally be used.

\subsection{Measures}

We gathered data about the usability of the demonstrators in comparison to conventional methods of connecting devices, using Bluetooth pairing. Here the usability is divided in three aspects; efficiency, effectiveness and satisfaction. The setup of the test was exploratory, but includes two proven methods to gain insight in the participants' mental models and have the participant score the usability, respectively the 'Teach-Back protocol' [15] and the 'System Usability Scale' [1. The action cycle by Norman [12] was also used to gain insight in the participants' mental models.

Added to these methods, we also collected data about task completion time, errors, recovery from errors and participants' satisfaction with using the method. A between-subjects design was used.

\subsection{Procedure}

Participants explored, made and broke connections between two mobile phones (a Nokia N95 and a Nokia XpressMusic), a sound system and an Ambient Light lamp. This was done using the Interaction Tile, Interaction Tabs and Bluetooth pairing.

Every session was recorded and notes were made by the moderator.

In this study, each participant worked through four phases of tasks starting with one of three methods (Interaction Tile, Interaction Tabs and Bluetooth pairing). Bluetooth pairing was tested as a comparative conventional method to measure the usability of the demonstrators.

Briefing. Participants received a brief explanation (5 min.) before the test, outside the 'Contextlab'. They were guided through the task path by the moderator. After the explanation, participants filled in a pre-test questionnaire and signed an informed consent form. The pre-test questionnaire included questions about: age, gender, occupation, self-report of familiarity with interactive systems (computers and mobile phones) and the participant's experience with usability studies and focus groups. 
Tasks. After the briefing, the participants worked on the actual tasks for about 30 minutes (including intermediary discussions). The task path for each method (Interaction Tile, Interaction Tabs and Bluetooth pairing) is as follows: First, users were introduced to the method and given three task descriptions. For each description they were asked to connect the devices or configure the demonstrator to perform the tasks (9 minutes). Second, users were given a task description and asked to fill in an Action Cycle diagram (6 minutes). Third, users were presented with three scenarios. For each scenario they were asked to explain which connections there were (9 minutes). Fourth, users were asked to explain what the method was they had used and how it worked using the teach-back protocol (6 minutes). The order of tasks was random but the same for each participant.

Debriefing. After the main tasks were performed there was a post-test questionnaire (5 minutes), where participants filled in the SUS questionnaire to rate the satisfaction of using the method. The session was concluded with a posttest discussion (5 minutes), where the moderator followed up on any particular problem that came up for the participant.

\subsection{Moderator Role}

The moderator sat in the room with the participant while conducting the session. The moderator introduced the session, conducted a short background interview, and then introduced tasks as appropriate. Because this study is somewhat exploratory, the moderator sometimes asked unscripted follow-up questions to clarify participants' behavior and expectations. The moderator also took notes and recorded the participants' behavior and comments. The session was digitally recorded on video using a Samsung NV8 digital camera.

\section{Results}

Unfortunately the system was not stable enough to accurately measure the performance data that was intended to be measured. The stability program also influenced the grades given by participants in the SUS questionnaire. Therefore the SUS scores were not reliable. However, we did gain certain insights from the observations and other measurements.

\subsection{Action Cycle Diagram}

The participants clearly had problems with filling in the Action Cycle Diagram. Only a few descriptions correspond to the predefined description. This can be explained by the fact that people do not consciously think about the seven steps as defined by Norman [12] during everyday activity. It is also not uncommon to go through several cycles before a goal is reached and not all of these cycles 
have to include all seven steps. This would require participants to fill in several diagrams or include several cycles in one diagram. Because this issue did not surface during the pilots or the first test, it would have been incorrect to change the procedure.

All participants followed roughly the same steps in achieving their goal. All but one participant forgot to mention the breaking of existing connections in 'Action specifications'. The participants using the Interaction Tabs and Bluetooth noticed this during the execution (before they thought they had achieved the goal) and went through another iteration immediately. Of the participants using the Interaction Tile, all but one participant noticed this after the execution (after they thought they had achieved the goal). These participants went through another iteration at a later stage but were also able to achieve their goal.

\subsection{Teach-Back Protocol}

While it is possible to draw conclusions concerning the actual mental models of participants, the protocol was mainly used to see if there were notable differences between the methods.

Although there were some differences between the participants individually, amongst the methods the drawings and explanations were roughly the same. None of the participants went into details about what happened in the background, but instead focused on the matters 'at hand'. Three participants (2 for the Interaction Tile and 1 for Bluetooth pairing) mentioned extending the current system with more devices (more mobile phones and a TV). One participant (Interaction Tile) was able to conclude that the connected devices were networked; the rest explained the connections in a hierarchical way.

In one of the examples given in 15 the researchers were able to conclude that participants tend to draw little when the system is transparent. If it is less transparent they are likely to make more detailed drawings to better support their story.

In this test the level of detail amongst the methods was roughly the same.

\subsection{Observations and Post-Test Discussion}

None of the participants that worked with the Interaction Tabs had trouble working with that method. During the post-test discussion they only wondered what was happening in the background. This was not because they had been unable to perform certain tasks, but because they suspected more was going on than what was visible to the user.

None of the participants working with Bluetooth pairing had trouble working with that method. They all mentioned that they were familiar with this way of connecting devices but had never experienced Bluetooth working this well.

The only real trouble for the participants working with the Interaction Tile was the initial experience with that method. It was not clear what the relation was between the center tile and the cubes and all four interpreted the pulsing 
green LED as a 'working connection'. One participant initially thought the LEDs were lasers that could 'read' the cubes when placed on top. Another participant thought it was only necessary to align the 'main' device to the center tile and align the other devices to the 'main' device.

During observations and post-test discussions it became clear that all but one participant were not able to get from the method that the connected devices were networked. The tasks given and the methods at hand led them to conclude that the connections were hierarchical and participants mainly followed one of two modes of arranging connections:

Linear (from one device to the next) - This was seen with the Interaction Tile and Interaction Tabs.

Centralized (from one device outwards) - This was seen with the Interaction Tabs and Bluetooth pairing.

Some participants sporadically arranged connections with the Interaction Tile in a way that indicated they took it for a network, but they explained verbally that they expected the system to make a hierarchy out of their arrangement. Some participants also explicitly mentioned that certain connections should not be possible while in fact they were.

\section{Discussion and Conclusion}

The most interesting results came from the observations and post-test discussions with the participants. The fact that all but one thought and worked in hierarchies is an interesting one. The Interaction Tile was designed to convey a different way of thinking, but instead participants projected their hierarchical way of thinking on the method. By making connections between no more than two devices at a time they did not use the full capacity of the system, took longer to perform the tasks and were slightly annoyed by the 'extra' work. Also, for those who thought in centralized hierarchies (one device in the center, the others around it), there was no way of projecting this thought on the Interaction Tile.

This is where the power of the Interaction Tabs showed, because it allows more ways of thinking (hierarchical, ontological, linear, and centralized). The participants found meaning in the arrangement of the tabs and the location of the tabs in relation to each other. For the system this does not matter; a connection is a connection and if devices are connected, they are networked.

This leads to conclude that the Interaction Tabs are a better fit for this scenario. Because of the setbacks, it is not possible to say whether the Interaction Tile and Interaction Tabs are better than Bluetooth pairing, although it appears that the Interaction Tabs are. It also appeared that participants were better able to perform the tasks with Bluetooth than with the Interaction Tile but this can be attributed to the fact that they had experience with Bluetooth pairing and connecting devices using a GUI.

For further research it would be interesting to see whether the hierarchical thinking of people can be generalized to scenarios other than the ones used in this user experiment. This could include other or more devices and media, or even 
completely different contexts. If it can be generalized, an interesting question would be whether solutions like the demonstrators should allow for hierarchical thinking while working with ontologies, or not.

Although the described experiment was useful and showed interesting results, it clearly has some limitations. For such an experiment to be successful, more participants are required, preferably without a design background. Six participants for each method is limited, four even more so. It is clearly not possible to collect reliable quantitative data with this number of participants.

Added to that, not all the methods used in the experiment worked out as expected. While the 'speak-out-loud' step of the Action Cycle diagram is useful to get insights in what participants think when performing tasks, the other steps seemed often unclear to them.

The results indicate that it may also be possible to elicit the mental model of the participants using the Teach-back protocol. It was useful for this test to see that all methods equally provided the participants with information, but the full potential of the protocol was not utilized.

If this user experiment were to be repeated at a later stage, the advice would be to have at least three people to be present during the tests; someone to manage the software and hardware, someone to guide the participant through the test and someone to make detailed notes. For a more qualitative approach, the fourth step of the Action Cycle diagram (think-out-loud) could be considered for each task. For a more quantitative approach, the Action Cycle diagram could be removed from the test completely, as well as the Teach-back protocol. This would allow for more tasks to be performed, which results in more data to be analyzed. A more elaborate usability questionnaire could be considered, although one has to take into account that lengthy questionnaires might annoy participants. This is especially to be considered when questionnaires are combined with performing tasks; this could lead to participants not paying enough attention when answering the questions.

\section{Acknowledgement}

SOFIA is funded by the European Artemis programme under the subprogramme SP3 Smart environments and scalable digital service. We would also like to thank the participants involved in the user experiment.

\section{References}

1. Brooke, J.: Sus-a quick and dirty usability scale. Usability Evaluation in Industry, pp. 189-194 (1996)

2. Dictionary: American heritage dictionary. The American Heritage Dictionary of the English Language (2000)

3. Feijs, L., Hu, J.: Component-wise mapping of media-needs to a distributed presentation environment. In: The 28th Annual International Computer Software and Applications Conference (COMPSAC 2004), pp. 250-257. IEEE Computer Society Press, Hong Kong (2004) 
4. Fitzmaurice, G., Ishii, H., Buxton, W.: Bricks: laying the foundations for graspable user interfaces, pp. 442-449. ACM Press/Addison-Wesley Publishing Co.

5. Hu, J.: Design of a Distributed Architecture for Enriching Media Experience in Home Theaters. Phd thesis, Department of Industrial Design, Eindhoven University of Technology (2006)

6. Hu, J., Feijs, L.: An adaptive architecture for presenting interactive media onto distributed interfaces. In: Hamza, M. (ed.) The 21st IASTED International Conference on Applied Informatics (AI 2003), pp. 899-904. ACTA Press, Innsbruck (2003)

7. Janse, M., van der Stok, P., Hu, J.: Distributing multimedia elements to multiple networked devices. In: User Experience Design for Pervasive Computing, Pervasive 2005, Munich, Germany (2005), http://www.fluidum.org/events/experience05

8. van Krannenburg, R.: The internet of things: A critique of ambient technology and the all-seeing network of rfid. Institute of Network Cultures (2008)

9. Laurillard, D.: Rethinking university teaching: A framework for the effective use of educational technology. Routledge, New York (1993)

10. Merrill, D., Kalanithi, J., Maes, P.: Siftables: towards sensor network user interfaces. In: TEI 2007, pp. 75-78. ACM, New York (2007)

11. Niezen, G., van der Vlist, B.J.J., Hu, J., Feijs, L.M.G.: From events to goals: Supporting semantic interaction in smart environments. In: 2010 IEEE Symposium on Computers and Communications (ISCC), Riccione, Italy, pp. 1029-1034 (2010)

12. Norman, D.: The design of everyday things. Basic Books, New York (2002)

13. SOFIA: Project website, http://www.sofia-project.eu/

14. Sterling, B.: Shaping things (2005)

15. van der Veer, G., Del Carmen Puerta Melguizo, M.: Mental models. In: The Human-Computer Interaction Handbook: Fundamentals, Evolving Technologies, and Emerging Applications, pp. 52-80 (2003)

16. van der Vlist, B., Niezen, G., Hu, J., Feijs, L.: Design semantics of connections in a smart home environment. In: Design and Semantics of Form and Movement (DeSForM 2010), Lucerne, Switzerland (2010) (accepted)

17. van der Vlist, B.J.J., Niezen, G., Hu, J., Feijs, L.M.G.: Semantic connections: Exploring and manipulating connections in smart spaces. In: 2010 IEEE Symposium on Computers and Communications (ISCC), Riccione, Italy, pp. 1-4 (2010)

18. Wensveen, S., Djajadiningrat, J., Overbeeke, C.: Interaction frogger: a design framework to couple action and function through feedback and feedforward. In: DIS 2004, pp. 177-184. ACM, New York (2004) 